ISSN 1855-3966 (printed edn.), ISSN 1855-3974 (electronic edn.)

\author{
ARS MATHEMATICA CONTEMPORANEA 16 (2019) 245-255 \\ https://doi.org/10.26493/1855-3974.1591.92e \\ (Also available at http://amc-journal.eu)
}

\title{
On the domination number and the total domination number of Fibonacci cubes
}

\author{
Elif Sayg1 * \\ Department of Mathematics and Science Education, Hacettepe University, \\ 06800, Beytepe, Ankara, Turkey
}

Received 2 February 2018, accepted 9 October 2018, published online 4 January 2019

\begin{abstract}
Fibonacci cubes are special subgraphs of the hypercube graphs. Their domination numbers and total domination numbers are obtained for some small dimensions by integer linear programming. For larger dimensions upper and lower bounds on these numbers are given. In this paper, we present the up-down degree polynomials for Fibonacci cubes containing the degree information of all vertices in more detail. Using these polynomials we define optimization problems whose solutions give better lower bounds on the domination numbers and total domination numbers of Fibonacci cubes. Furthermore, we present better upper bounds on these numbers.
\end{abstract}

Keywords: Fibonacci cubes, domination number, total domination number, integer linear programming.

Math. Subj. Class.: 05C69, 68R10, $11 B 39$

\section{Introduction}

Let $G=(V(G), E(G))$ be a graph with vertex set $V(G)$ and edge set $E(G)$. $D \subseteq V(G)$ is a dominating set of $G$ if every vertex in $V(G)$ either belongs to $D$ or is adjacent to some vertex in $D$. The domination number $\gamma(G)$ is defined as the minimum cardinality of a dominating set of the graph $G$. Similarly, $D \subseteq V(G)$ is a total dominating set if every vertex in $V(G)$ is adjacent to some vertex in $D$ and the total domination number $\gamma_{t}(G)$ is defined as the minimum cardinality of a total dominating set of $G$. Note that the total domination number is defined for isolate-free graphs and it is not defined for the graphs that contain isolated vertices. The domination number of the Fibonacci cubes $\Gamma_{n}$ is first given

\footnotetext{
* Supported by TÜBİTAK under Grant No. 117R032. The author would like to thank the anonymous reviewers for their valuable comments and would like to thank Prof. Dr. Ömer Eğecioğlu for useful discussions and suggestions.

E-mail address: esaygi@hacettepe.edu.tr (Elif Sayg1)
} 
in [14] and [2]. These results are extended in [8] by using integer linear programming for some cases. Total domination number of $\Gamma_{n}$ is considered in [1], in which an upper bound and a lower bound on $\gamma_{t}\left(\Gamma_{n}\right)$ are obtained. The exact values of $\gamma\left(\Gamma_{n}\right)$ and $\gamma_{t}\left(\Gamma_{n}\right)$ are also considered by using integer programming in [1]. The upper bound on $\gamma_{t}\left(\Gamma_{n}\right)$ given in [1] is improved in [15]. We summarize these results in Section 2. The aim of this work is to improve some of the results given in [1] and [15].

The hypercube $Q_{n}$ of dimension $n \geq 1$ is the graph with vertex set $V\left(Q_{n}\right)=\{0,1\}^{n}$, in which two vertices are adjacent if they differ in one coordinate. For convenience $Q_{0}=$ $K_{1}$. All the vertices of $Q_{n}$ are labeled by the binary strings of length $n$. The Fibonacci cubes $\Gamma_{n}$ are special subgraphs of $Q_{n}$ and they were introduced by Hsu [7] as a model of interconnection networks. In literature, many interesting properties of the Fibonacci cubes have been investigated, see survey [9] for details. In recent years results on disjoint hypercubes in $\Gamma_{n}$ are presented in $[5,13,16]$ and the cube enumerator polynomial of $\Gamma_{n}$ is considered in $[10,11,17]$ and many combinatorial results are given. The domination-type invariants of $\Gamma_{n}$ are considered in [1, 2, 8, 14, 15] and some numerical results and bounds are presented.

It is known that Fibonacci strings of length $n$ are the binary strings of length $n$ that contain no consecutive ones. For this reason we can write

$$
\begin{aligned}
& V\left(\Gamma_{n}\right)=\left\{b_{1} b_{2} \cdots b_{n} \mid b_{i} \in\{0,1\}, 1 \leq i \leq n, \text { and } b_{i} \cdot b_{i+1}=0 \text { for } 1 \leq i<n\right\} \text { and } \\
& E\left(\Gamma_{n}\right)=\left\{(u, v) \mid u, v \in V\left(\Gamma_{n}\right), d_{H}(u, v)=1\right\},
\end{aligned}
$$

where $d_{H}(u, v)$ denotes the Hamming distance between $u$ and $v$, that is, the number of different coordinates in $u$ and $v$. The number of vertices of the Fibonacci cubes $\Gamma_{n}$ is $F_{n+2}$, where $F_{n}$ are the Fibonacci numbers defined as $F_{0}=0, F_{1}=1$ and $F_{n}=F_{n-1}+F_{n-2}$ for $n \geq 2$. For $n \geq 2$ we will use the following formulation for the fundamental decomposition of $\Gamma_{n}$ (see, [9]):

$$
\Gamma_{n}=0 \Gamma_{n-1}+10 \Gamma_{n-2} .
$$

Here note that $0 \Gamma_{n-1}$ is the subgraph of $\Gamma_{n}$ induced by the vertices that start with 0 and $10 \Gamma_{n-2}$ is the subgraph of $\Gamma_{n}$ induced by the vertices that start with 10 . Furthermore, $0 \Gamma_{n-1}$ has a subgraph isomorphic to $00 \Gamma_{n-2}$, and there is a matching between $00 \Gamma_{n-2}$ and $10 \Gamma_{n-2}$ (see Figure 1).
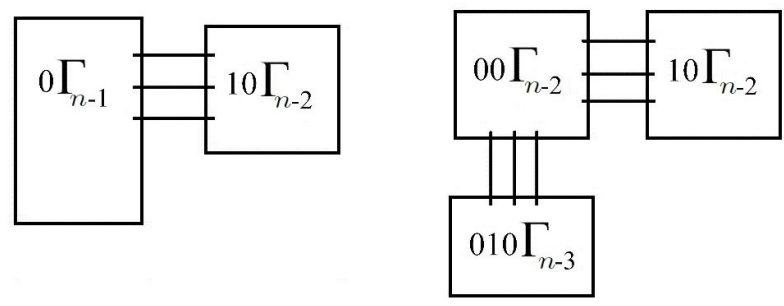

Figure 1: Fundamental decompositions of the Fibonacci cube $\Gamma_{n}, n \geq 3$.

In this paper, we present upper bounds on $\gamma\left(\Gamma_{n}\right)$ and $\gamma_{t}\left(\Gamma_{n}\right)$. Furthermore, we introduce the up-down degree polynomials for $\Gamma_{n}$ containing the degree information of all vertices $V\left(\Gamma_{n}\right)$ in more detail. Using these polynomials we define optimization problems whose solutions give lower bound on $\gamma\left(\Gamma_{n}\right)$ and $\gamma_{t}\left(\Gamma_{n}\right)$. 


\section{Known results and new upper bounds on $\gamma\left(\Gamma_{n}\right)$ and $\gamma_{t}\left(\Gamma_{n}\right)$}

In this section, first we summarize some known results on the domination number and the total domination number of Fibonacci cubes and then we present new uppper bounds for these numbers. We start with Figure 2 and Figure 3 showing a dominating set and a total dominating set for small dimensional $\Gamma_{n}$ 's.
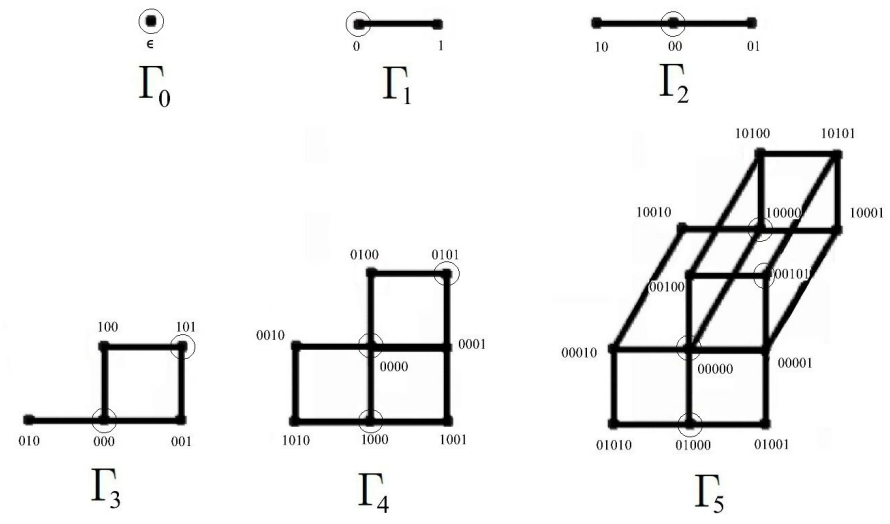

Figure $2: \Gamma_{0}, \ldots, \Gamma_{5}$ and their dominating sets.
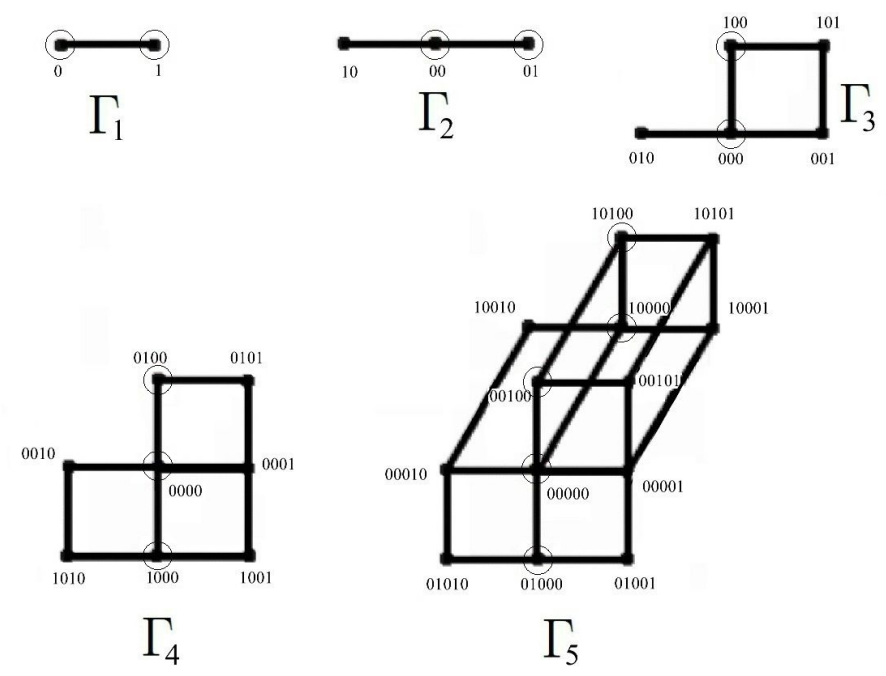

Figure 3: $\Gamma_{1}, \ldots, \Gamma_{5}$ and their total dominating sets.

We collect the known values of $\gamma\left(\Gamma_{n}\right)$ and $\gamma_{t}\left(\Gamma_{n}\right)$ in Table 1 . The values of $\gamma\left(\Gamma_{n}\right)$ for $n \leq 8$ are obtained in [14]. The other values of $\gamma\left(\Gamma_{n}\right)$ are obtained by integer programming. For $n=9$ and $n=10$ they are obtained in [8] and for $n=11$ and $n=12$ they are obtained in [1]. Similarly, all the values of $\gamma_{t}\left(\Gamma_{n}\right)$ given in Table 1 are obtained by computer using integer programming in [1]. 
Table 1: Known values of $\gamma\left(\Gamma_{n}\right)$ and $\gamma_{t}\left(\Gamma_{n}\right)$.

\begin{tabular}{c|c|c|c|c|c|c|c|c|c|c|c|c|c}
$n$ & 1 & 2 & 3 & 4 & 5 & 6 & 7 & 8 & 9 & 10 & 11 & 12 & 13 \\
\hline \hline$\left|V\left(\Gamma_{n}\right)\right|$ & 2 & 3 & 5 & 8 & 13 & 21 & 34 & 55 & 89 & 144 & 233 & 377 & 610 \\
\hline \hline$\gamma\left(\Gamma_{n}\right)$ & 1 & 1 & 2 & 3 & 4 & 5 & 8 & 12 & 17 & 25 & 39 & $54-61$ & \\
\hline$\gamma_{t}\left(\Gamma_{n}\right)$ & 2 & 2 & 2 & 3 & 5 & 7 & 10 & 13 & 20 & 30 & 44 & 65 & $97-101$
\end{tabular}

Now we describe the integer linear programming used in [8] and [1]. Suppose each vertex $v \in V\left(\Gamma_{n}\right)$ is associated with a binary variable $x_{v}$. Let $N(v)$ be the set of vertices adjacent to $v$ and $N[v]=N(v) \cup\{v\}$. The problems of determining $\gamma\left(\Gamma_{n}\right)$ and $\gamma_{t}\left(\Gamma_{n}\right)$ can be expressed as a problem of minimizing the objective function

$$
\sum_{v \in V\left(\Gamma_{n}\right)} x_{v}
$$

subject to the condition that for every $v \in V\left(\Gamma_{n}\right)$ we have

$$
\begin{aligned}
& \sum_{a \in N[v]} x_{a} \geq 1 \text { (for domination number), } \\
& \sum_{a \in N(v)} x_{a} \geq 1 \text { (for total domination number). }
\end{aligned}
$$

The value of the objective function is then $\gamma\left(\Gamma_{n}\right)$ and $\gamma_{t}\left(\Gamma_{n}\right)$ respectively. Note that this problem has $F_{n+2}$ variables and $F_{n+2}$ constraints. In [1], it is stated that $\gamma\left(\Gamma_{12}\right)$ and $\gamma_{t}\left(\Gamma_{13}\right)$ were not computed in real time using the above optimization problem. They got the estimates

$$
54 \leq \gamma\left(\Gamma_{12}\right) \leq 61 \text { and } 97 \leq \gamma_{t}\left(\Gamma_{13}\right) \leq 101
$$

Here, the main difficulty is the order of $\Gamma_{n}$ which equals to the number of variables and the number of constraints.

By using the degree information of the vertices in $\Gamma_{n}$ the following lower bound on $\gamma\left(\Gamma_{n}\right)$ is presented in [14].

Theorem 2.1 ([14]). If $n \geq 9$, then

$$
\gamma\left(\Gamma_{n}\right) \geq\left\lceil\frac{F_{n+2}-2}{n-2}\right\rceil .
$$

By using a similar technique the following lower bound on $\gamma_{t}\left(\Gamma_{n}\right)$ is obtained in [1].

Theorem 2.2 ([1]). If $n \geq 9$, then

$$
\gamma_{t}\left(\Gamma_{n}\right) \geq\left\lceil\frac{F_{n+2}-11}{n-3}\right\rceil-1
$$

In Section 3 we propose an optimization problem having less number of variables and constraints to estimate lower bounds on $\gamma\left(\Gamma_{n}\right)$ and $\gamma_{t}\left(\Gamma_{n}\right)$. Our results improve the lower 
bounds given in Theorem 2.1 and Theorem 2.2 and we present some numerical values in Table 2 and Table 3.

By using the exact values in Table 1 and the fundamental decomposition (1.1) of $\Gamma_{n}$, the following upper bound on $\gamma_{t}\left(\Gamma_{n}\right)$ is obtained in [1].

Theorem 2.3 ([1]). If $n \geq 11$, then $\gamma_{t}\left(\Gamma_{n}\right) \leq 21 F_{n-8}+2 F_{n-10}$.

In [1], using the computer result $\gamma_{t}\left(\Gamma_{13}\right) \leq 101$ the upper bound in Theorem 2.3 improved to

$$
\gamma_{t}\left(\Gamma_{n}\right) \leq 601 F_{n-1}-371 F_{n}, \quad n \geq 12 .
$$

These two upper bounds further improved in [15] by using the values of $\gamma\left(\Gamma_{n}\right)$ and the fundamental decomposition (1.1) of $\Gamma_{n}$ more than once.

Theorem 2.4 ([15]). If $n \geq 15$, then

$$
\begin{aligned}
\gamma\left(\Gamma_{n}\right) & \leq \gamma_{t}\left(\Gamma_{n}\right) \leq 3 \gamma\left(\Gamma_{n-3}\right)+2 \gamma\left(\Gamma_{n-4}\right) \\
& \leq 116 F_{n}-187 F_{n-1}=21 F_{n-8}-\left(2 F_{n-10}+F_{n-12}\right) .
\end{aligned}
$$

Furthermore, $\gamma_{t}\left(\Gamma_{14}\right) \leq 166$.

We implemented the same integer linear programming problem (2.1) using CPLEX in NEOS Server [3, 4, 6] for $n=13$ and obtain the estimates (takes approximately 2 hours)

$$
78 \leq \gamma\left(\Gamma_{13}\right) \leq 93
$$

Using this result with $\gamma\left(\Gamma_{12}\right) \leq 61$ we obtain the following bound on the domination number of $\Gamma_{n}$.

Theorem 2.5. If $n \geq 12$, then $\gamma\left(\Gamma_{n}\right) \leq 21 F_{n-8}-\left(2 F_{n-10}+8 F_{n-12}\right)$.

Proof. The proof mimics the proof of [1, Theorem 2.1]. By the fundamental decomposition (1.1) of $\Gamma_{n}$ we have $\gamma\left(\Gamma_{n}\right) \leq \gamma\left(\Gamma_{n-1}\right)+\gamma\left(\Gamma_{n-2}\right)$. We know that $\gamma\left(\Gamma_{12}\right) \leq 61$ and $\gamma\left(\Gamma_{13}\right) \leq 93$. For $n \geq 12$ define the sequence $\left(b_{n}\right)$ with $b_{n}=b_{n-1}+b_{n-2}$ where $b_{12}=61$ and $b_{13}=93$. Then by induction we have $b_{n}=21 F_{n-8}-2 F_{n-10}-8 F_{n-12}$ for any $n \geq 12$. We complete the proof since $\gamma\left(\Gamma_{n}\right) \leq b_{n}$ for $n \geq 12$.

Combining the results in Theorem 2.5 and Theorem 2.4 we get the following result which improves Theorem 2.3 and Theorem 2.4.

Theorem 2.6. If $n \geq 16$, then

$$
\gamma_{t}\left(\Gamma_{n}\right) \leq 21 F_{n-8}-\left(2 F_{n-10}+8 F_{n-12}\right) .
$$

\section{Up-down degree enumerator polynomial}

In this section we present the up-down degree enumerator polynomial for $\Gamma_{n}$. It contains the degree information of all vertices $V\left(\Gamma_{n}\right)$ in more detail. Using this polynomial we write optimization problems whose solutions are lower bounds on $\gamma\left(\Gamma_{n}\right)$ and $\gamma_{t}\left(\Gamma_{n}\right)$.

For each fixed $v \in V\left(\Gamma_{n}\right)$ we write a monomial $x^{u} y^{d}$ where $d=w(v)$ is the Hamming weight of $v$ and $u$ is $\operatorname{deg}(v)-d$ (that is, $\operatorname{deg}(v)=u+d$ ). Recall that, by the definition of $\Gamma_{n},\left(v, v^{\prime}\right) \in E\left(\Gamma_{n}\right)$ if and only if $d_{H}\left(v, v^{\prime}\right)=1$. Therefore, the number of neighbors of $v$ 
whose weight is one more than the weight of $v$ (say up neighbors of $v, w\left(v^{\prime}\right)=w(v)+1$ ) is $u$ and the number of neighbors of $v$ whose weight is one less than the weight of $v$ (say down neighbors of $v, w\left(v^{\prime}\right)=w(v)-1$ ) is $d$. For this reason we call the polynomial

$$
P_{n}(x, y)=\sum_{v \in V\left(\Gamma_{n}\right)} x^{\operatorname{deg}(v)-w(v)} y^{w(v)}=\sum_{v \in V\left(\Gamma_{n}\right)} x^{u} y^{d}
$$

as the up-down degree enumerator polynomial of $\Gamma_{n}$. By using the fundamental decomposition (1.1) of $\Gamma_{n}$ we obtain the following recursive relation which will be useful to calculate $P_{n}(x, y)$.

Theorem 3.1. Let $P_{n}(x, y)$ be the up-down degree enumerator polynomial of $\Gamma_{n}$. Then for $n \geq 3$ we have

$$
P_{n}(x, y)=x P_{n-1}(x, y)+y P_{n-2}(x, y)+y P_{n-3}(x, y)-x y P_{n-3}(x, y)
$$

where

$$
P_{0}(x, y)=1, \quad P_{1}(x, y)=x+y \quad \text { and } \quad P_{2}(x, y)=x^{2}+2 y .
$$

Proof. $P_{0}, P_{1}$ and $P_{2}$ are clear from Figure 2. Assume that $n \geq 3$. We know that the updown degree enumerator polynomials of $\Gamma_{n-1}, \Gamma_{n-2}$ and $\Gamma_{n-3}$ are $P_{n-1}(x, y), P_{n-2}(x, y)$ and $P_{n-3}(x, y)$ respectively. By $(1.1)$ we have

$$
\begin{aligned}
\Gamma_{n} & =0 \Gamma_{n-1}+10 \Gamma_{n-2} \\
& =\left(00 \Gamma_{n-2}+010 \Gamma_{n-3}\right)+10 \Gamma_{n-2}
\end{aligned}
$$

and there is a matching between $00 \Gamma_{n-2}$ and $10 \Gamma_{n-2}$ (see also Figure 1). From this decomposition we have the following three different cases:

1. Assume that $v \in 10 \Gamma_{n-2}$. These vertices are the ones in $\Gamma_{n-2}$ whose weights $d=w(v)$ increase by one in $\Gamma_{n}$. Furthermore, their degrees increase by one due to the matching between $00 \Gamma_{n-2}$ and $10 \Gamma_{n-2}$, which means that $u=\operatorname{deg}(v)-w(v)$ remains the same in $\Gamma_{n}$. Therefore, these vertices contribute $y P_{n-2}(x, y)$ to $P_{n}(x, y)$.

2. Assume that $v \in 010 \Gamma_{n-3}$. These vertices are the ones in $\Gamma_{n-3}$ whose weights $d=w(v)$ increase by one in $\Gamma_{n}$ and their degrees increase by one due to the matching between $010 \Gamma_{n-3}$ and $000 \Gamma_{n-3} \subset 00 \Gamma_{n-2}$, which means that $u=\operatorname{deg}(v)-w(v)$ remains the same in $\Gamma_{n}$. Therefore, these vertices contribute $y P_{n-3}(x, y)$ to $P_{n}(x, y)$.

3. Assume that $v \in 00 \Gamma_{n-2}$. These vertices are the ones in $0 \Gamma_{n-1}$ that are not in $010 \Gamma_{n-3}$. In $\Gamma_{n-1}$ the up-down degree enumerator polynomial of these vertices becomes $P_{n-1}(x, y)-y P_{n-3}(x, y)$. The weights $d=w(v)$ of all such vertices remain the same in $\Gamma_{n}$ but their degrees increase by one due to the matching between $00 \Gamma_{n-2}$ and $10 \Gamma_{n-2}$, that is, $u=\operatorname{deg}(v)-w(v)$ increase by one in $\Gamma_{n}$. Therefore, these vertices contribute $x\left(P_{n-1}(x, y)-y P_{n-3}(x, y)\right)$ to $P_{n}(x, y)$.

By adding all of the above contributions we get the desired result.

Now we describe an optimization problem using the up-down degree enumerator polynomial $P_{n}(x, y)$. Let $D_{T}$ be a total dominating set of $\Gamma_{n}$. Then by the definition of Fibonacci cubes for every vertex $v \in V\left(\Gamma_{n}\right)$ with weight $w(v)$ then there must exist a vertex 
$v_{D} \in N(v) \cap D_{T}$ with $w\left(v_{D}\right)=w(v) \mp 1$. Furthermore, assume that for any fixed vertex $v_{D} \in D_{T}$ its corresponding monomial be $x^{u} y^{d}$ in the $P_{n}(x, y)$. This means that $v_{D}$ dominates $u$ distinct vertices $v \in V\left(\Gamma_{n}\right)$ with weight $w(v)=w\left(v_{D}\right)+1$ and $d$ distinct vertices $v \in V\left(\Gamma_{n}\right)$ with weight $w(v)=w\left(v_{D}\right)-1$. Note that for all $v_{D} \in D_{T}$ some of the dominated vertices may coincide. Now assume that

$$
P_{n}(x, y)=\sum_{v \in V\left(\Gamma_{n}\right)} x^{u} y^{d}=\sum c_{d}^{u} x^{u} y^{d}
$$

For each pair $(u, d)$ in $P_{n}(x, y)$ we associate an integer variable $z_{d}^{u}$ which counts the number of vertices in $D_{T}$ with weight $d$ and degree $u+d$, that is, the number of vertices in $D_{T}$ having $d$ down neighbors and $u$ up neighbors. Clearly, we have the bounds $0 \leq z_{d}^{u} \leq c_{d}^{u}$. Our aim is to minimize $\left|D_{T}\right|$, that is, our objective function is to minimize

$$
\sum_{u, d} z_{d}^{u}
$$

Then by the above observation to dominate all the vertices having a fixed weight $d$ such that $1 \leq d \leq\left\lceil\frac{n}{2}\right\rceil-1$ we must have

$$
r_{d}: \sum_{u}\left(u \cdot z_{d-1}^{u}+(d+1) \cdot z_{d+1}^{u}\right) \geq \sum_{u} c_{d}^{u}
$$

since any vertex with weight $d-1$ having $u$ up neighbors can dominate $u$ distinct vertices with weight $d$ and any vertex with weight $d+1$ (all have $d+1$ down neighbors) can dominate $d+1$ distinct vertices with weight $d$. By the same argument, for $d=0$ we must have

$$
r_{0}: \sum_{u} z_{1}^{u} \geq \sum_{u} c_{0}^{u}=1
$$

and for $d=\left\lceil\frac{n}{2}\right\rceil$ we must have

$$
r_{\left\lceil\frac{n}{2}\right\rceil}: \sum_{u} u \cdot z_{\left\lceil\frac{n}{2}\right\rceil-1}^{u} \geq \sum_{u} c_{\left\lceil\frac{n}{2}\right\rceil}^{u}= \begin{cases}1 & \text { if } n \text { is odd, } \\ \frac{n}{2}+1 & \text { if } n \text { is even. }\end{cases}
$$

Now subject to these constraints $r_{0}, \ldots, r_{\left\lceil\frac{n}{2}\right\rceil}$ the value of the objective function will be a lower bound on $\gamma_{t}\left(\Gamma_{n}\right)$. Similarly, to find a lower bound on $\gamma\left(\Gamma_{n}\right)$ we need to modify all of the constraints $r_{d}, 0 \leq d \leq\left\lceil\frac{n}{2}\right\rceil$. By the definition of the dominating set, for each fixed $d$ we need to add all of the variables $z_{d}^{u}$ to the left side of the constraint $r_{d}$.

Remark 3.2. We remark that using [12, Theorem 4.6] we can easily obtain the coefficients $c_{d}^{u}$ in (3.3). By the definition of the up-down degree enumerator polynomial we know that $c_{d}^{u}$ is the number of vertices in $\Gamma_{n}$ whose number of up neighbors is $u$ and weight is $d$. That is, $c_{d}^{u}$ equals to the number of vertices of $\Gamma_{n}$ having degree $u+d$ and weight $d$. Therefore, [12, Theorem 4.6] gives

$$
c_{d}^{u}=\left(\begin{array}{c}
d+1 \\
n-2 d-u+1
\end{array}\right)\left(\begin{array}{c}
n-2 d \\
u
\end{array}\right) .
$$


Remark 3.3. We know that the number of vertices of $\Gamma_{n}$ with weight $d$ is equal to the right hand side of the above constraints $r_{d}$. By definition of $\Gamma_{n}$ this number is equal to the number of Fibonacci strings of length $n$ and weight $d$. Therefore we have

$$
\sum_{u} c_{d}^{u}=\left(\begin{array}{c}
n-d+1 \\
d
\end{array}\right) .
$$

Remark 3.4. To find the number of variables $z_{d}^{u}$ we need to find the number of monomials in $P_{n}(x, y)$. Assume that $n$ is even. Then by the structure of the vertices in Fibonacci cubes (it can also be seen from the structure of Fibonacci strings) $n-3 d \leq u \leq n-2 d$. Therefore the number of variables $z_{d}^{u}$ becomes

$$
\sum_{d=0}^{\left\lfloor\frac{n}{3}\right\rfloor}(d+1)+\sum_{d=\left\lfloor\frac{n}{3}\right\rfloor+1}^{\frac{n}{2}}(n-2 d+1)
$$

which is equal to

$$
s^{2}-2 s r+\frac{3 r(r+1)}{2}+1
$$

where $r=\left\lfloor\frac{n}{3}\right\rfloor$ and $s=n / 2$. Similarly, if $n$ is odd we obtain that the number of variables $z_{d}^{u}$ is

$$
s^{2}-s(2 r+1)+\frac{r(3 r+5)}{2}+2
$$

where $r=\left\lfloor\frac{n}{3}\right\rfloor$ and $s=\left\lceil\frac{n}{2}\right\rceil$.

Now we illustrate our optimization problem for $n=14$. We have the following polynomial by Theorem 3.1.

$$
\begin{aligned}
P_{14}(x, y)= & 8 y^{7}+ \\
& 7 y^{6} x^{2}+42 y^{6} x+35 y^{6}+ \\
& 6 y^{5} x^{4}+60 y^{5} x^{3}+120 y^{5} x^{2}+60 y^{5} x+6 y^{5}+ \\
& 5 y^{4} x^{6}+60 y^{4} x^{5}+150 y^{4} x^{4}+100 y^{4} x^{3}+15 y^{4} x^{2}+ \\
& 4 y^{3} x^{8}+48 y^{3} x^{7}+112 y^{3} x^{6}+56 y^{3} x^{5}+ \\
& 3 y^{2} x^{10}+30 y^{2} x^{9}+45 y^{2} x^{8}+ \\
& 2 y x^{12}+12 y x^{11}+ \\
& x^{14}
\end{aligned}
$$

and this polynomial corresponds to the following optimization problem:

\section{Objective function:}

$$
\begin{aligned}
\min : & z_{0}^{14}+z_{1}^{12}+z_{1}^{11}+z_{2}^{10}+z_{2}^{9}+z_{2}^{8}+z_{3}^{8}+z_{3}^{7}+z_{3}^{6}+z_{3}^{5}+ \\
& z_{4}^{6}+z_{4}^{5}+z_{4}^{4}+z_{4}^{3}+z_{4}^{2}+z_{5}^{4}+z_{5}^{3}+z_{5}^{2}+z_{5}^{1}+z_{5}^{0}+z_{6}^{2}+z_{6}^{1}+z_{6}^{0}+z_{7}^{0}
\end{aligned}
$$


Constraints for $\gamma_{t}\left(\Gamma_{14}\right)$ :

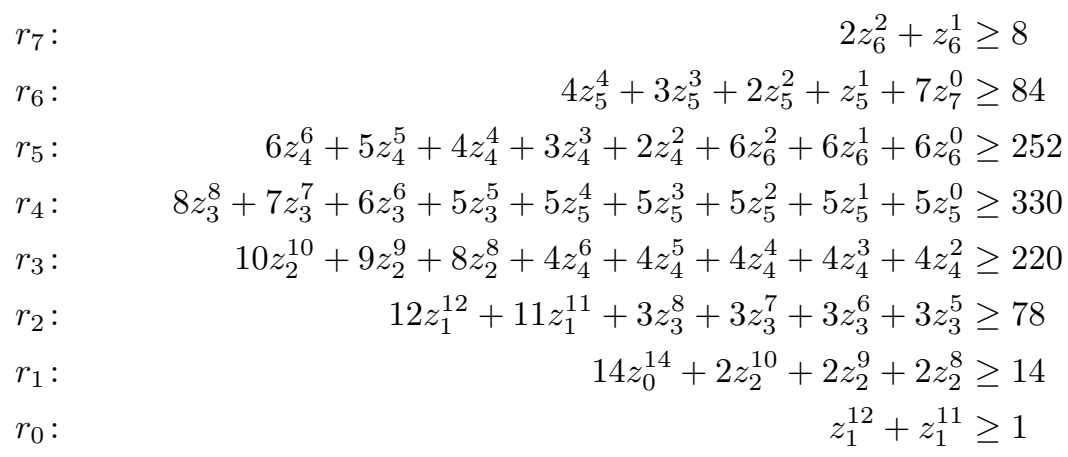

Constraints for $\gamma\left(\Gamma_{14}\right)$ :

$$
\begin{array}{cc}
r_{7}: & 2 z_{6}^{2}+z_{6}^{1}+z_{7}^{0} \geq 8 \\
r_{6}: & 4 z_{5}^{4}+3 z_{5}^{3}+2 z_{5}^{2}+z_{5}^{1}+z_{6}^{2}+z_{6}^{1}+z_{6}^{0}+7 z_{7}^{0} \geq 84 \\
r_{5}: & 6 z_{4}^{6}+5 z_{4}^{5}+4 z_{4}^{4}+3 z_{4}^{3}+2 z_{4}^{2}+z_{5}^{4}+z_{5}^{3}+ \\
& z_{5}^{2}+z_{5}^{1}+z_{5}^{0}+6 z_{6}^{2}+6 z_{6}^{1}+6 z_{6}^{0} \geq 252 \\
r_{4}: & 8 z_{3}^{8}+7 z_{3}^{7}+6 z_{3}^{6}+5 z_{3}^{5}+z_{4}^{6}+z_{4}^{5}+z_{4}^{4}+z_{4}^{3}+ \\
& z_{4}^{2}+5 z_{5}^{4}+5 z_{5}^{3}+5 z_{5}^{2}+5 z_{5}^{1}+5 z_{5}^{0} \geq 330 \\
r_{3}: & 10 z_{2}^{10}+9 z_{2}^{9}+8 z_{2}^{8}+z_{3}^{8}+z_{3}^{7}+z_{3}^{6}+ \\
z_{3}^{5}+4 z_{4}^{6}+4 z_{4}^{5}+4 z_{4}^{4}+4 z_{4}^{3}+4 z_{4}^{2} \geq 220 \\
r_{2}: & 12 z_{1}^{12}+11 z_{1}^{11}+z_{2}^{10}+z_{2}^{9}+z_{2}^{8}+3 z_{3}^{8}+3 z_{3}^{7}+3 z_{3}^{6}+3 z_{3}^{5} \geq 78 \\
r_{1}: & 14 z_{0}^{14}+z_{1}^{12}+z_{1}^{11}+2 z_{2}^{10}+2 z_{2}^{9}+2 z_{2}^{8} \geq 14 \\
r_{0}: & z_{0}^{14}+z_{1}^{12}+z_{1}^{11} \geq 1
\end{array}
$$

Bounds:

$$
\begin{aligned}
& z_{0}^{14} \leq 1 \quad z_{1}^{12} \leq 2 \quad z_{1}^{11} \leq 12 \quad z_{2}^{10} \leq 3 \quad z_{2}^{9} \leq 30 \\
& z_{2}^{8} \leq 45 \quad z_{3}^{8} \leq 4 \quad z_{3}^{7} \leq 48 \quad z_{3}^{6} \leq 112 \quad z_{3}^{5} \leq 56 \\
& z_{4}^{6} \leq 5 \quad z_{4}^{5} \leq 60 \quad z_{4}^{4} \leq 150 \quad z_{4}^{3} \leq 100 \quad z_{4}^{2} \leq 15 \\
& z_{5}^{4} \leq 6 \quad z_{5}^{3} \leq 60 \quad z_{5}^{2} \leq 120 \quad z_{5}^{1} \leq 60 \quad z_{5}^{0} \leq 6 \\
& z_{6}^{2} \leq 7 \quad z_{6}^{1} \leq 42 \quad z_{6}^{0} \leq 35 \quad z_{7}^{0} \leq 8
\end{aligned}
$$

The value of the objective function gives a lower bound on $\gamma\left(\Gamma_{14}\right)$ and $\gamma_{t}\left(\Gamma_{14}\right)$ respectively. Note that the above problem have only 24 variables and 8 constraints (instead of having 987 variables and 987 constraints, see Section 2). In general using the up-down degree enumerator polynomial $P_{n}(x, y)$ of $\Gamma_{n}$ in Theorem 3.1 we can write an optimization problem having less number of variables $z_{d}^{u}$ (see Remark 3.4) and $\left\lceil\frac{n}{2}\right\rceil+1$ constraints $r_{d}$. The solutions of these problem give lower bounds on $\gamma\left(\Gamma_{n}\right)$ and $\gamma_{t}\left(\Gamma_{n}\right)$. One can easily see that the number of variables and the number of constraints are very smaller than the ones in the optimization problem described in Section 2. 
For illustration we implemented the above integer linear programming problem using CPLEX in NEOS Server [3, 4, 6] for $13<n \leq 26$ and immediately obtain the lower bounds presented in Table 2 and Table 3. Note that for $n=26$, the number of variables in our optimization problem is 70 by Remark 3.4 and it is $F_{28}=317811$ for the general optimization problem (2.1). In addition, the upper bounds in these tables are obtained by Theorem 2.5 for $n \geq 14$ and Theorem 2.6 for $n \geq 16$. Note that the first bounds in both tables are obtained in [1] and the upper bounds on $\gamma_{t}\left(\Gamma_{14}\right)$ and $\gamma_{t}\left(\Gamma_{15}\right)$ comes from Theorem 2.4.

Table 2: Current best bounds on $\gamma\left(\Gamma_{n}\right), 12 \leq n \leq 26$.

\begin{tabular}{c|c||c|c||c|c}
$n$ & $\gamma\left(\Gamma_{n}\right)$ & $n$ & $\gamma\left(\Gamma_{n}\right)$ & $n$ & $\gamma\left(\Gamma_{n}\right)$ \\
\hline \hline 12 & $54-61$ & 17 & $344-648$ & 22 & $3060-7189$ \\
\hline 13 & $78-93$ & 18 & $528-1049$ & 23 & $4748-11632$ \\
\hline 14 & $98-154$ & 19 & $819-1697$ & 24 & $7381-18821$ \\
\hline 15 & $148-247$ & 20 & $1270-2746$ & 25 & $11472-30453$ \\
\hline 16 & $224-401$ & 21 & $1970-4443$ & 26 & $17912-49274$
\end{tabular}

Table 3: Current best bounds on $\gamma_{t}\left(\Gamma_{n}\right), 13 \leq n \leq 26$.

\begin{tabular}{c|c||c|c||c|c}
$n$ & $\gamma\left(\Gamma_{n}\right)$ & $n$ & $\gamma\left(\Gamma_{n}\right)$ & $n$ & $\gamma\left(\Gamma_{n}\right)$ \\
\hline \hline 13 & $97-101$ & 18 & $578-1049$ & 23 & $5075-11632$ \\
\hline 14 & $110-166$ & 19 & $890-1697$ & 24 & $7865-18821$ \\
\hline 15 & $164-261$ & 20 & $1374-2746$ & 25 & $12191-30453$ \\
\hline 16 & $246-401$ & 21 & $2121-4443$ & 26 & $19033-49274$ \\
\hline 17 & $376-648$ & 22 & $3281-7189$ & &
\end{tabular}

Remark 3.5. For $n=12$, Theorem 2.1 gives $\gamma\left(\Gamma_{12}\right) \geq 38$ and Theorem 2.2 gives $\gamma_{t}\left(\Gamma_{12}\right) \geq 40$. The values of the objective function in our optimization problems having 19 variables and 7 constraints give lower bounds $\gamma\left(\Gamma_{12}\right) \geq 44$ and $\gamma_{t}\left(\Gamma_{12}\right) \geq 50$.

For the case $n=13$, Theorem 2.1 gives $\gamma\left(\Gamma_{13}\right) \geq 56$ and Theorem 2.2 gives $\gamma_{t}\left(\Gamma_{13}\right) \geq 59$. The values of the objective function in our optimization problems having 22 variables and 8 constraints give lower bounds $\gamma\left(\Gamma_{13}\right) \geq 65$ and $\gamma_{t}\left(\Gamma_{13}\right) \geq 75$.

\section{References}

[1] J. Azarija, S. Klavžar, Y. Rho and S. Sim, On domination-type invariants of Fibonacci cubes and hypercubes, Ars Math. Contemp. 14 (2018), 387-395, doi:10.26493/1855-3974.1172.bae.

[2] A. Castro, S. Klavžar, M. Mollard and Y. Rho, On the domination number and the 2-packing number of Fibonacci cubes and Lucas cubes, Comput. Math. Appl. 61 (2011), 2655-2660, doi:10.1016/j.camwa.2011.03.012.

[3] J. Czyzyk, M. P. Mesnier and J. J. Moré, The NEOS server, IEEE Comput. Sci. Eng. 5 (1998), 68-75, doi:10.1109/99.714603. 
[4] E. D. Dolan, NEOS Server 4.0 Administrative Guide, Technical Memorandum ANL/MCS-TM250, Mathematics and Computer Science Division, Argonne National Laboratory, May 2001.

[5] S. Gravier, M. Mollard, S. Špacapan and S. S. Zemljič, On disjoint hypercubes in Fibonacci cubes, Discrete Appl. Math. 190/191 (2015), 50-55, doi:10.1016/j.dam.2015.03.016.

[6] W. Gropp and J. J. Moré, Optimization environments and the NEOS server, in: M. D. Buhmann and A. Iserles (eds.), Approximation Theory and Optimization, Cambridge University Press, Cambridge, pp. 167-182, 1997, selected papers from the Conference on Numerical Mathematics, in honor of M. J. D. Powell on the occasion of his 60th birthday, held in Cambridge, July $27-30,1996$.

[7] W.-J. Hsu, Fibonacci cubes-a new interconnection topology, IEEE Trans. Parallel Distrib. Syst. 4 (1993), 3-12, doi:10.1109/71.205649.

[8] A. Ilić and M. Milošević, The parameters of Fibonacci and Lucas cubes, Ars Math. Contemp. 12 (2017), 25-29, doi:10.26493/1855-3974.915.f48.

[9] S. Klavžar, Structure of Fibonacci cubes: a survey, J. Comb. Optim. 25 (2013), 505-522, doi: 10.1007/s10878-011-9433-z.

[10] S. Klavžar and M. Mollard, Cube polynomial of Fibonacci and Lucas cubes, Acta Appl. Math. 117 (2012), 93-105, doi:10.1007/s10440-011-9652-4.

[11] S. Klavžar and M. Mollard, Daisy cubes and distance cube polynomial, European J. Combin. (2018), doi:10.1016/j.ejc.2018.02.019.

[12] S. Klavžar, M. Mollard and M. Petkovšek, The degree sequence of Fibonacci and Lucas cubes, Discrete Math. 311 (2011), 1310-1322, doi:10.1016/j.disc.2011.03.019.

[13] M. Mollard, Non covered vertices in Fibonacci cubes by a maximum set of disjoint hypercubes, Discrete Appl. Math. 219 (2017), 219-221, doi:10.1016/j.dam.2016.10.029.

[14] D. A. Pike and Y. Zou, The domination number of Fibonacci cubes, J. Combin. Math. Combin. Comput. 80 (2012), 433-444.

[15] E. Sayg1, Upper bounds on the domination and total domination number of fibonacci cubes, SDU J. Nat. Appl. Sci. 21 (2017), 782-785, doi:10.19113/sdufbed.05851.

[16] E. Sayg1 and Ö. Eğecioğlu, Counting disjoint hypercubes in Fibonacci cubes, Discrete Appl. Math. 215 (2016), 231-237, doi:10.1016/j.dam.2016.07.004.

[17] E. Saygı and Ö. Eğecioğlu, $q$-cube enumerator polynomial of Fibonacci cubes, Discrete Appl. Math. 226 (2017), 127-137, doi:10.1016/j.dam.2017.04.026. 